\title{
Diffraction inspired unidirectional and bidirectional beam splitting in defect-containing photonic structures without interface corrugations
}

Evrim Colak, Andriy E. Serebryannikov, P. V. Usik, and Ekmel Ozbay

Citation: Journal of Applied Physics 119, 193108 (2016); doi: 10.1063/1.4949509

View online: https://doi.org/10.1063/1.4949509

View Table of Contents: http://aip.scitation.org/toc/jap/119/19

Published by the American Institute of Physics

\section{Articles you may be interested in}

Acoustic metasurface-based perfect absorber with deep subwavelength thickness

Applied Physics Letters 108, 063502 (2016); 10.1063/1.4941338

Unidirectional transmission using array of zero-refractive-index metamaterials

Applied Physics Letters 104, 193509 (2014); 10.1063/1.4878400

Effect of in-material losses on terahertz absorption, transmission, and reflection in photonic crystals made of polar dielectrics

Journal of Applied Physics 118, 133101 (2015); 10.1063/1.4932017

Unidirectional acoustic transmission through a prism with near-zero refractive index

Applied Physics Letters 103, 053505 (2013); 10.1063/1.4817249

Acoustic energy harvesting using resonant cavity of a sonic crystal

Applied Physics Letters 95, 013506 (2009); 10.1063/1.3176019

Asymmetric acoustic transmission through near-zero-index and gradient-index metasurfaces

Applied Physics Letters 108, 223502 (2016); 10.1063/1.4953264

\section{PHYSICS TODAY}

MANAGER'S GUIDE

WHITEPAPERS

\section{READ NOW}

PRESENTED BY

Accelerate R\&D with

Multiphysics Simulation
○OMSOL 


\title{
Diffraction inspired unidirectional and bidirectional beam splitting in defect-containing photonic structures without interface corrugations
}

\author{
Evrim Colak, ${ }^{1}$ Andriy E. Serebryannikov, ${ }^{2, a)}$ P. V. Usik, ${ }^{3}$ and Ekmel Ozbay ${ }^{4}$ \\ ${ }^{1}$ Electrical Engineering Department, Ankara University, Golbasi, 06830 Ankara, Turkey \\ ${ }^{2}$ Faculty of Physics, Adam Mickiewicz University, 61-614 Poznań, Poland \\ ${ }^{3}$ Institute of Radio Astronomy, National Academy of Sciences of Ukraine, 61002 Kharkiv, Ukraine \\ ${ }^{4}$ Nanotechnology Research Center-NANOTAM, Bilkent University, 06800 Ankara, Turkey
}

(Received 20 December 2015; accepted 2 May 2016; published online 20 May 2016)

\begin{abstract}
It is shown that strong diffractions and related dual-beam splitting can be obtained at transmission through the nonsymmetric structures that represent two slabs of photonic crystal $(\mathrm{PhC})$ separated by a single coupled-cavity type defect layer, while there are no grating-like corrugations at the interfaces. The basic operation regimes include unidirectional and bidirectional splitting that occur due to the dominant contribution of the first positive and first negative diffraction orders to the transmission, which is typically connected with different manifestations of the asymmetric transmission phenomenon. Being the main component of the resulting transmission mechanism, diffractions appear owing to the effect exerted by the defect layer that works like an embedded diffractive element. Two mechanisms can co-exist in one structure, which differ, among others, in that whether dispersion allows coupling of zero order to a wave propagating in the regular, i.e., defectfree $\mathrm{PhC}$ segments or not. The possibility of strong diffractions and efficient splitting related to it strongly depend on the dispersion properties of the Floquet-Bloch modes of the PhC. Existence of one of the studied transmission scenarios is not affected by location of the defect layer. Published by AIP Publishing. [http://dx.doi.org/10.1063/1.4949509]
\end{abstract}

\section{INTRODUCTION}

Unidirectional transmission of electromagnetic waves ${ }^{1,2}$ and beam splitting ${ }^{3-5}$ belong to the functionalities, which are widely used and highly demanded in practical devices working in different frequency ranges from microwaves to the visible. Recently, an approach has been suggested that enables combination of these two functionalities in one Lorentzreciprocal device when two opposite incidence directions and rather arbitrary directions of the outgoing beams (which differ from the incidence direction) are allowed. ${ }^{6}$ Its realization with the aid of a finite-thickness slab of two-dimensional photonic crystal $(\mathrm{PhC})$ with the rods made of isotropic, passive, linear dielectric material, and one-side corrugations has been demonstrated for the so-called direct regime of asymmetric transmission. ${ }^{6,7}$ In this case, the ability of directional selectivity is connected with the common effect of diffractions arising due to the one-side interface corrugations and a specific, i.e., anisotropic type or ultralow-index isotropic type dispersion of the $\mathrm{PhC}$, at which zero-order transmission is blocked, whereas some of higher diffraction orders may be transmitted but only at the corrugated-side incidence. ${ }^{7}$ On the other hand, strong asymmetry in transmission and related asymmetry in beam splitting can be obtained due to corrugations when dispersion permits coupling of zero order at the noncorrugated incidence interface (but it is suppressed in transmission), i.e., in the inverse regime. ${ }^{8}$ In any case, placing corrugations at the interfaces has been the main route to obtain such diffractions that are required for asymmetric transmission.

${ }^{\text {a)} E l e c t r o n i c ~ m a i l: ~ a n d s e r @ a m u . e d u . p l . ~}$
Asymmetric transmission is a very general phenomenon, which can be connected not only with diffractions at the interfaces $^{6-11}$ but also with polarization conversion, ${ }^{12-15}$ direction manipulation by inclining interfaces like in prism-shaped configurations, ${ }^{16-19}$ etc. Metamaterials may promise more compact performances for devices based on diffraction inspired asymmetric transmission, ${ }^{20-22}$ but their application to splitting has not yet been studied. Moreover, it can be obtained in transmission through thin corrugated structures with a slit ${ }^{23,24}$ and in-plane propagation of surface waves. ${ }^{25}$ The common feature of all reciprocal devices based on asymmetric transmission is that the spatial inversion symmetry is broken. Clearly, they would not enable the same functionality as that obtainable in the nonreciprocal framework, but at the same time may enable such types of directional selectivity that are useful for practical devices. In fact, asymmetric transmission can be considered as the generalized mode conversion, which is sensitive to the change of the incidence direction for the opposite one. ${ }^{26,27}$ One should also mention planar metamaterials (metasurfaces) enabling beam manipulation, e.g., by using the phase discontinuity approach. ${ }^{28-31}$

In this paper, we show that strong diffractions and splitting inspired by them can be obtained in nonsymmetric photonic structures without interface corrugations but with an embedded diffractive element, which can be used as parts of complex devices and circuits based on PhCs. The main goal of this study is to demonstrate the principal possibility and richness of both unidirectional and bidirectional splitting regimes which are achievable for the studied class of the structures in a wide range of parameter variation. We study transmission in slabs of square-lattice rod-type dielectric 
$\mathrm{PhC}$ with the embedded coupled-cavity type defect layer that is composed of the same rods as the regular part of the structure, which can be used to obtain splitting. The suitable diffractions are created by the periodic defect layer that distinguishes the studied structures from those proposed earlier for asymmetric transmission and unidirectional splitting. The defect layer may, in principle, contribute to realization of other functions (e.g., those directly connected with its defect like nature like a defect-mode waveguide ${ }^{32,33}$ ) in other frequency ranges in the same structure. This is especially promising from the multifunctional operation perspective. It will be demonstrated that both direct and inverse regimes of asymmetric transmission, with or without coupling of zero order to a wave propagating in the regular part of $\mathrm{PhC}$, can yield efficient splitting. The possibility of obtaining of various splitting regimes in the frequency ranges, which correspond to different Floquet-Bloch modes of the $\mathrm{PhC}$ and, thus, to different dispersion types, will be analyzed. The main attention will be paid to the transmission results, whereas dispersion results are used for the additional explanations of the observed coupling scenarios. At the same time, a figure-of-merit type quantifying of the splitting regimes would be not practical, because a sole criterium is ambiguous and requirements for the concrete device or circuit should be taken into account that is beyond the scope of this paper. To obtain transmission results, a flexible iterative solver based on the coupled integral equation technique is used; it has controllable convergence, which can be accelerated by preconditioning. ${ }^{34}$ Dispersion is calculated using CST Microwave Studio, a commercial solver based on finite integration technique. ${ }^{35}$

\section{GEOMETRY AND GENERAL PRINCIPLES}

Figure 1 presents the general geometry of the studied problem and schematics that illustrate the possible roles of different parts of the entire nonsymmetric structure and appropriate splitting scenarios. The studied nonsymmetric structures represent a slab that consists of two segments of two-dimensional square-lattice $\mathrm{PhC}$ with lattice constant $a$ (denoted by $A$ and $C$ ) and a coupled-cavity type defect layer located between these two segments (denoted by $B$ ). It is assumed that the segment $A$ contains a smaller number of the rod layers than the segment $C$. The structure is illuminated by a normally incident $s$-polarized electromagnetic wave (the electric field vector is along the rod axes) at the angle of incidence $\theta=0$, from either the upper or the lower side. Consideration is restricted here to the case when defects in the segment $B$ are obtained by taking a single regular layer of the rods (like those in the segments $A$ and $C$ ), and then removing every second rod so that the period along $x$-axis is $L=2 a$. In fact, it determines the periodicity of the entire structure, which is assumed to be infinite in the $x$-direction.

A difference in number of the layers within the segments $A$ and $C$ is required in order to fulfill the requirement of broken spatial inversion symmetry, without which asymmetry in transmission and, hence, unidirectional splitting cannot be achieved. ${ }^{7,11}$ We consider a frequency range corresponding to low-order Floquet-Bloch modes of PhC, to which higher

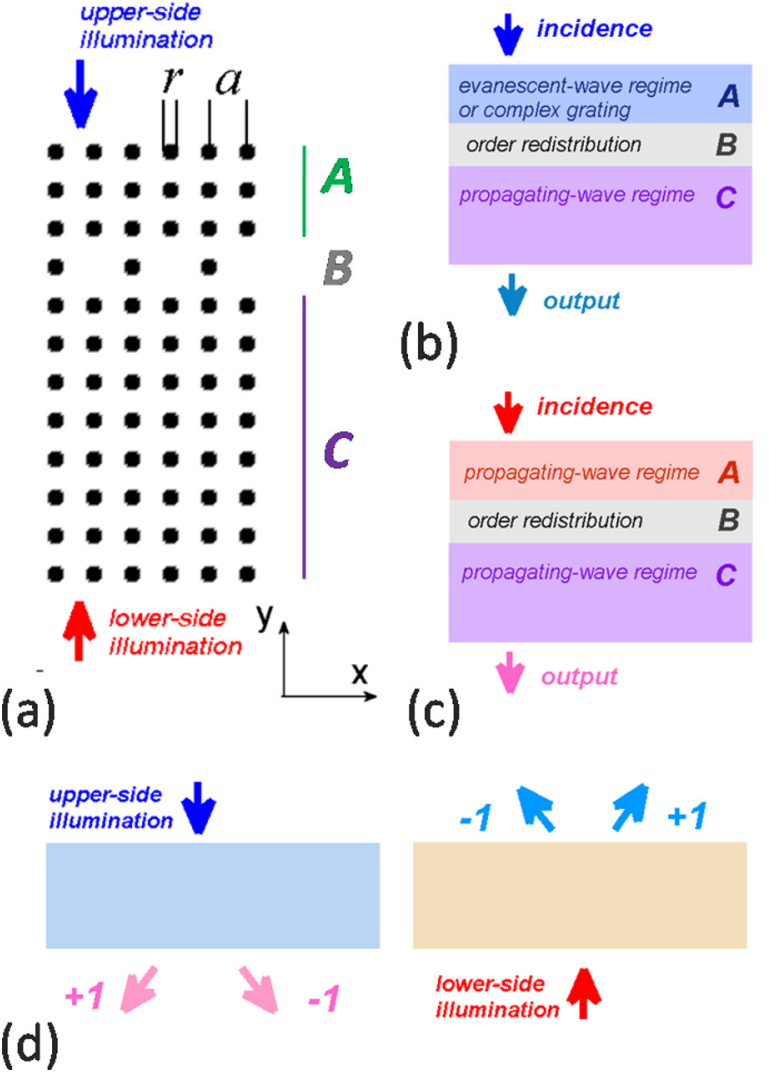

FIG. 1. (a) General geometry of the finite-thickness slab of PhC with a defect layer; schematics illustrating the roles of the defect layer at upperside illumination when zero order is either (b) not coupled or (c) coupled at the upper interface, and (d) schematics showing the basic features of the ideal diffraction inspired splitting at (left panel) upper-side illumination and (right panel) lower-side illumination.

diffraction orders $(|m|>0)$ cannot be coupled when the interface period is $a$.

Depending on whether the order $m=0$ can be coupled at the interfaces or not, two main groups of the transmission scenarios can be distinguished. The difference is schematically demonstrated in Figs. 1(b) and 1(c). If zero order can be coupled, the both segments $A$ and $C$ are in the propagatingwave regime, while a properly designed layer $B$ might create redistribution of the wave energy in favor of higher orders, which are also allowed to propagate in the regular segments but cannot be coupled at $\theta=0$ when the period is $a$. If zero order cannot be in-coupled, we obtain evanescent-wave regime in the segment $A$ (at the upper-side illumination), while the defect layer can transform evanescent waves into ones allowed to propagate in the regular segments due to the higher orders $(|m|>0)$. If the segment $A$ contains just one or two layers of the rods, evanescence might be weakly pronounced so that the segments $A$ and $B$ should be considered rather like a part of one complex grating, which does not have interface corrugations. Note that Figs. 1(b) and 1(c) correspond to the case when the segment $A$ is adjusted to the incidence interface. In case when the segment $C$ is illuminated, the segments $A$ and $C$ are formally in propagating-wave and evanescent-wave regimes, respectively, for the scenario similar to Fig. 1(b), and both segments are in propagating-wave regime for the scenario similar to Fig. 1(c). 
Splitting can appear due to diffraction orders $m= \pm 1$, while zero order has to be suppressed in transmission, even if it is efficiently in-coupled. Then, if transmission is fully blocked at the opposite-side illumination, unidirectional transmission takes place. In this case, zero order should not be in-coupled, or in-coupled but insignificantly contributing to the transmission due to a specific adjustment of structural parameters. In the ideal case of unidirectional splitting, one should obtain either

$$
T^{\rightarrow}=\overrightarrow{t_{-1}}+\overrightarrow{t_{+1}} \quad \text { and } \quad T^{\leftarrow}=0
$$

or

$$
T^{\leftarrow}=t_{-1}^{\leftarrow}+t_{+1}^{\leftarrow} \quad \text { and } \quad T^{\rightarrow}=0,
$$

where $T^{\rightarrow}$ and $T^{\leftarrow}$ mean total transmittance at the upper-side and the lower-side illumination, respectively; $t_{ \pm 1}^{\rightarrow}$ and $t_{ \pm 1}^{\leftarrow}$ mean partial \pm 1 -order transmittance (diffraction efficiency) at the upper-side and the lower-side illumination. The conditions given by Eqs. (1) and (2) correspond to the direct regime and the inverse regime of the diffraction inspired unidirectional transmission, respectively. To avoid ambiguity in the use of these regime names, we should note that there is no full analogy with the structures having external corrugations. ${ }^{8}$ However, it is expected that the main transmission features can be similar so that the use of this terminology is quite reasonable.

These two regimes correspond to the schematics shown in the left and the right panel in Fig. 1(d). Earlier, it has been shown for a slab of $\mathrm{PhC}$ with one-side corrugations that zero order can be well (although not perfectly) suppressed, even if being in-coupled. ${ }^{8}$ It is worth noting that the symmetry properties result in that $\underset{-1}{\overrightarrow{ }}=\underset{+1}{\rightarrow}$ and $t_{-1}^{\leftarrow}=t_{+1}^{\leftarrow}$, according to the general grating theory, ${ }^{36}$ whereas the reciprocity results in that $t_{0}=t_{0}^{\rightarrow}=t_{0}^{\leftarrow}$. This means that dual-beam splitting will appear if zero-order transmission is suppressed $\left(t_{0}=0\right)$, whereas the orders with $|m|=1$ may propagate due to the segment $B$ but those with $|m|>1$ remain evanescent. ${ }^{6,8}$

In turn, splitting can be bidirectional, i.e., both $T^{\rightarrow}$ and $T^{\leftarrow}$ are quite high mainly due to the contribution of the orders $m= \pm 1$. However, it is difficult to obtain exactly bidirectional dual-beam splitting with $T^{\rightarrow}=T^{\leftarrow}$ and $t_{+1}^{\rightarrow}$ $=t_{ \pm 1}^{\leftarrow}$ in a nonsymmetric structure. Furthermore, suppression of the propagating zero order can be problematic. Clearly, enhancement or suppression of the corresponding counterparts of the resulting physical mechanism may yield a more suitable configuration, e.g., in terms of efficiency. Throughout the paper, we assume that the total number of the rod layers in the segments $A, B$, and $C$ is $N=12$.

\section{RESULTS AND DISCUSSION}

\section{A. Basic effects}

Now, we analyze the results of numerical study that are aimed to prove that the expected transmission and splitting regimes can really appear in $\mathrm{PhC}$ based structures with an embedded diffractive element. Figure 2 presents transmittance vs $k L(k=\omega / c)$ for four nonsymmetric structures,

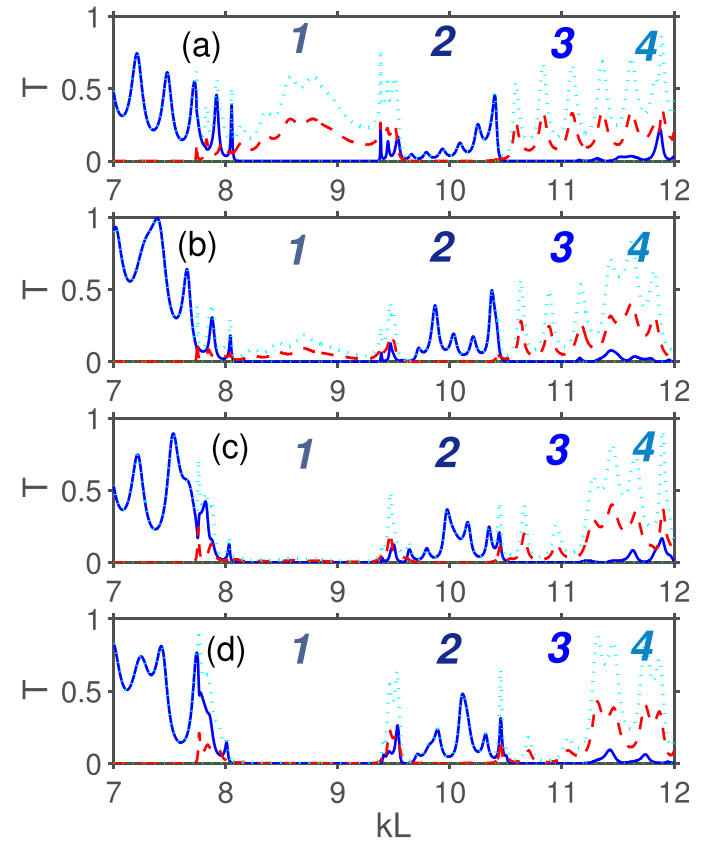

FIG. 2. Transmittance vs $k L$ for structure with defect layer being (a) second, (b) third, (c) fourth, and (d) fifth one from the upper (here incidence) interface; $d / a=0.4, \varepsilon=5.8$; solid blue line $t_{0}$, dashed red line $t_{-1}^{\rightarrow}$ and $t_{+1}$, dotted cyan line $T^{\rightarrow}$.

which differ from each other in location of the coupledcavity type defect layer. The value of $\varepsilon=5.8$ is chosen here that corresponds to the range to which some types of glass and diamond do belong. In the $k L$-ranges where transmission is forbidden at $\theta=0$ for the corresponding uniform (i.e., defect-free) slab of $\mathrm{PhC}$, the field can still be strong at the second layer location. Thus, in contrast with evanescence expected to appear when the segment $A$ contains several regular layers of the rods, the most upper regular layer can be considered in this case just as a part of the complex grating with internal corrugations. Then, coupling to propagating waves due to higher diffraction orders and the resulting transmission might be quite strong. Indeed, one can see in Fig. 2(a) two such $k L$-ranges with $T^{\rightarrow} \approx t_{-1}^{\rightarrow}+t_{+1}$, which are denoted by 1 and 3 . They are located at $8.1<k L<9.4$ and $10.6<k L<11.0$, respectively. Since $\overrightarrow{t_{-1}}=\overrightarrow{t_{+1}}$, these ranges are suitable for dual-beam splitting, although optimization is needed to obtain higher efficiency.

Two more ranges of interest are denoted by 2 and 4 . They are located at $9.4<k L<10.6$ and $k L>11.0$, where zero order contributes to $T^{\rightarrow}$. In the range 2 , we obtain $t_{-1}=0$, and hence, $T^{\rightarrow}=t_{0}$. In the range $4, \overrightarrow{t_{-1}}>0$, and thus, $T^{\rightarrow}=t_{0}+t_{-1}+t_{+1}$ in the general case. In a larger part of this range, diffractions are strong, i.e., $t_{-1}+t_{+1} \gg t_{0}$. As a result, dual-beam splitting can be obtained that is imperfect because of the contribution of $t_{0}$. In fact, a sole irregular internal layer may fully change the transmission scenario as compared to the uniform slab of $\mathrm{PhC}$ with the same $N$, for which either perfect reflection (ranges 1 and 3 ) or zero-order transmission (ranges 2 and 4 ) could only be possible at $\theta=0$ (not shown).

Next, let us shift the defect layer downward so that it becomes the third and, then, the fourth layer from the upper 
interface, see Figs. 2(b) and 2(c). This means that the number of the rod layers of the segment $A$ being in the evanescentwave regime is increased, leading to the decrease of $T^{\rightarrow}$ within the $k L$-ranges denoted by 1 and 3 . However, the extent of decrease for these ranges is different, being connected with different properties of Floquet-Bloch modes and, thus, with different coupling conditions. For the range 4, one should notice a weak effect of zero order, strong diffractions, and the related potential in splitting. These features remain whatever the depth of the defect layer embedding is. This gives big freedom in design, i.e., the defect layer may be involved in realization of another functionality (not considered here), which requires a deep embedding of this layer. ${ }^{32,33}$

In Fig. 2(d), transmittance is shown for the structure, in which the defect layer is the fifth one from the upper interface, while the segment $C$ contains seven regular layers. For such a structure, evanescent waves may be strongly damped when zero order is not coupled at the upper incidence interface. Now, $T^{\rightarrow} \approx 0$ in the range 1 . In the range 3 , in-coupling to the evanescent waves and their transformation into the propagating waves at the segment $B$ results in non-vanishing transmission. For instance, $T^{\rightarrow} \approx t_{-1}+t_{+1}$ exceeds 0.12 and 0.09 at $10.6<k L<11$ in Figs. 2(c) and 2(d), respectively.

One more interesting feature is observed at the right edge of the range 2 in Figs. 2(c) and 2(d), where significant contribution of the orders $m= \pm 1$ to $T^{\rightarrow}$ occurs near $k L=10.45$, at which $t_{-1} \approx 0$ and $t_{+1} \approx 0$ in Fig. 2(a). Thus, contribution of the orders $m= \pm 1$ can strongly depend on the thicknesses of the segments $A$ and $C$.

To further explain the specifics of the coupling scenarios realized in the ranges 1-4 in Fig. 2 and predict behavior of $T^{\leftarrow}$, Fig. 3 presents the equifrequency dispersion contours (EFCs) plotted in the wave vector plane by using the repeated zone diagram approach, ${ }^{7,37}$ for four selected $k L$ -
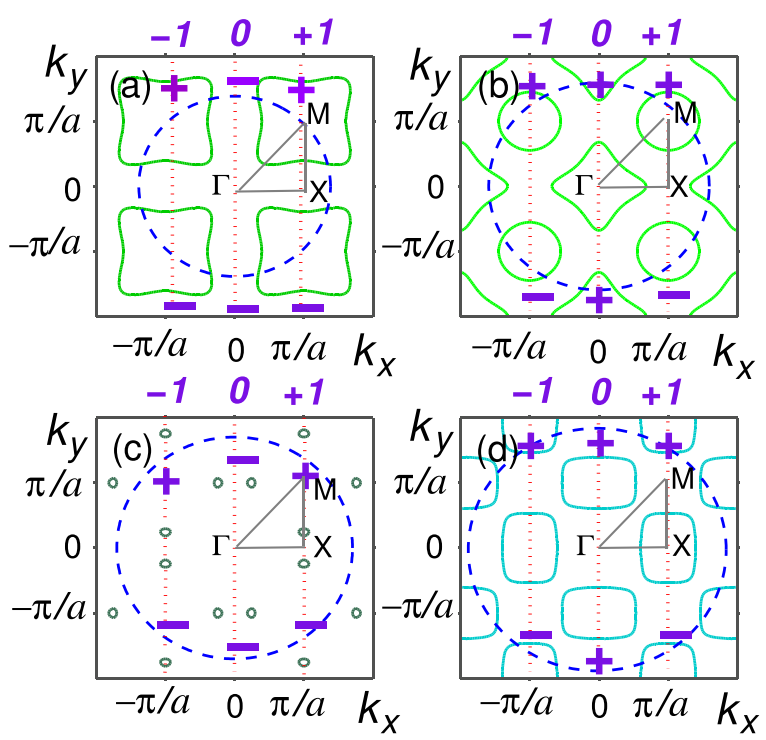

FIG. 3. EFCs in $\left(k_{x}, k_{y}\right)$-plane for the regular infinite PhC with $d / a=0.4$ and $\varepsilon=5.8$ (solid lines) and surrounding air (dashed lines) at (a) $k L=8.7$, (b) $k L=10$, (c) $k L=10.7$, and (d) $k L=11.7$. Construction lines (vertical dotted lines) are located in line with Eqs. (4) and (5); they are denoted by $-1,0$, and +1 according to their order $(m)$. Signs + and - indicate that coupling of the corresponding order is possible or impossible, respectively; signs at plot top and plot bottom correspond to the periods of $P=L=2 a$ and $P=a$. values. The eigenmode solver of CST Microwave Studio ${ }^{35}$ was used to calculate dispersion as $k_{x}$ vs $\omega$ for a large number of the fixed values of $k_{y}$; then, a self-made code is utilized to combine these results so that a standard MATLAB function can be used for plotting isolines. This allows us to present dispersion results in a form like in Fig. 3. Each $k_{x^{-}}$ value within the range limited by a circular contour (plotted for surrounding air) corresponds to a certain value of $\theta$, i.e.,

$$
k_{x}=k \sin \theta,
$$

where $k=\omega / c$. If an EFC exists for the $\mathrm{PhC}$ at a value of $k_{x}$ given by Eq. (3), this means that zero order is coupled to a wave propagating in the PhC. In turn, if an EFC exists for a value of $k_{x}$ that is given by

$$
k_{x}=k \sin \phi_{m}
$$

where

$$
\sin \phi_{m}=\sin \theta+2 \pi m /(k L),
$$

then the $m$-th order is coupled at the period $L$. In particular, at $\theta=0$, it occurs if an EFC exists at

$$
\sin \phi_{ \pm 1}= \pm 2 \pi /(k L) .
$$

Figure 3(a) shows the situation arising in the range 1 in Fig. 2, when the order $m=0$ is not coupled but the orders $m= \pm 1$ are coupled, provided that $P=L=2 a$. The latter are not coupled at $P=a$. Hence, the role of the segment $B$ in obtaining of the propagating-wave regime in the regular part of $\mathrm{PhC}$ is evident. The difference in coupling at $P=a$ and $P=2 a$, which is illustrated in Fig. 3(a), is necessary for obtaining unidirectional transmission. According to Fig. 3(b), zero order may be coupled in the range 2 at $P=a$, whereas the appearance of propagating waves due to the \pm 1 -orders invokes a larger period, e.g., $P=2 a$. For the range 2 , we obtain good agreement between Figs. 2 and 3(b). The same remains true regarding the range 3 in Figs. 2 and 3(c). In this case, incoupling due to zero order is impossible at $\theta=0$ but possible at $\left|k_{x}\right| / k \approx 0.6$ due to the \pm 1 -orders. Again, coupling cannot appear at $P=a$ but can appear at $P=2 a$. Finally, we compare the range 4 in Figs. 2 and 3(d). Formally, coupling scenario in Fig. 3(d) is the same as in Fig. 3(b), whereas contribution of the orders $m=0, \pm 1$ to $T^{\rightarrow}$ in the ranges 2 and 4 in Fig. 2 is substantially different.

Figure 4 presents transmittance vs $k L$ for the same four structures as in Fig. 2 but at lower-side illumination. Thus, Fig. 4(a) corresponds to the case when the defect layer is the eleventh one from the incidence interface. For such a thick segment $C$, evanescent-wave regime prevents significant transmission, as occurs in the ranges 1 and 3. In turn, zeroorder in-coupling and transmission may appear in the studied nonsymmetric structure at $\theta=0$ within pass bands of the uniform $\mathrm{PhC}$, which correspond to the ranges 2 and 4, while the defect layer redistributes energy between the different diffraction orders. In fact, this transmission scenario is similar to that studied in Ref. 8 for uniform slabs of $\mathrm{PhC}$ with corrugations at one of the interfaces. However, the difference 

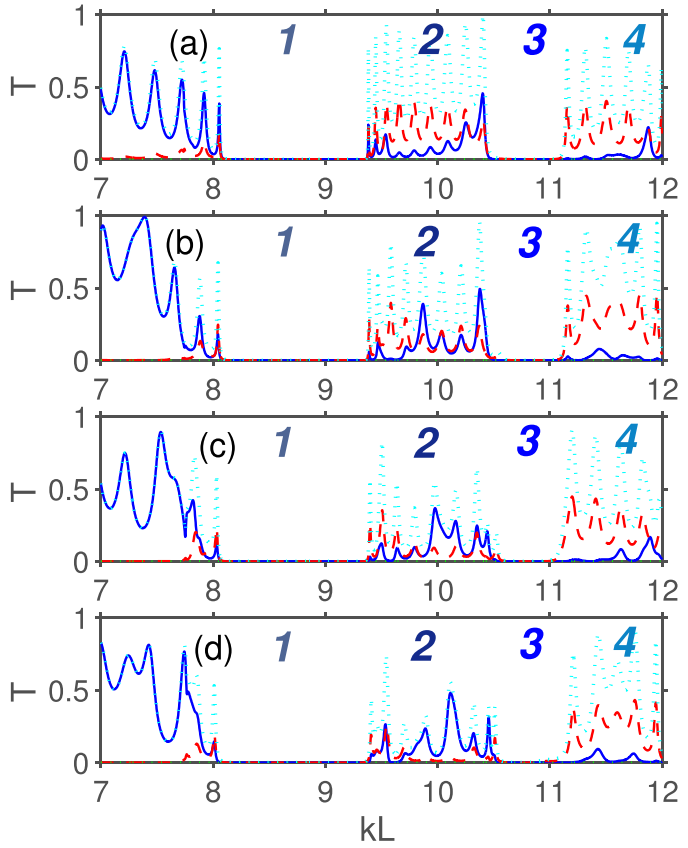

FIG. 4. Transmittance vs $k L$ for structure with defect layer being (a) second, (b) third, (c) fourth, and (d) fifth one from the upper (here exit) interface; $d / a$ $=0.4, \varepsilon=5.8$; solid blue line $t_{0}$, dashed red line $t_{-1}^{\leftarrow}$ and $t_{+1}^{\leftarrow}$, dotted cyan line $T^{\leftarrow}$.

from Ref. 8 is that now we have two segments, $A$ and $C$, in propagating-wave regime.

Let us compare the ranges 1 and 3 in Figs. 2 and 4 from the point of view of asymmetry in transmission. In Figs. 2(a) and 4(a), one can see that $T^{\rightarrow}=\overrightarrow{t_{-1}}+\overrightarrow{t_{+1}}>0$ and $T^{\leftarrow} \approx 0$. Shifting the defect layer one period lower [see Figs. 2(b) and 4(b)], two periods lower [see Figs. 2(c) and 4(c)], and, finally, four periods lower [see Figs. 2(d) and 4(d)] does not affect the strong damping that remains in the ranges 1 and 3 in Figs. 4(a)-4(d). However, well-pronounced asymmetry in transmission and related unidirectional dual-beam splitting can be obtained in these ranges in the direct regime $\left(T^{\rightarrow}>0\right.$ and $T^{\leftarrow}=0$ ) only when the defect layer is rather close to the upper interface.

Next, we consider the ranges 2 and 4 in Figs. 2 and 4. Although contribution of $t_{0}$ to $T^{\rightarrow}$ and $T^{\leftarrow}$ is possible, the role of higher orders can be dominant and, thus, diffraction inspired dual-beam splitting can still be realized. In these ranges, in-coupling can be obtained due to zero order, with redistribution of the wave energy in favor of the \pm 1 -orders occurring at the segment $B$. In the range 2, the effect of location of the defect layer on the achievable values of $T^{\leftarrow}$ is significant. Since $t_{-1}^{\rightarrow} \approx 0$ [except for vicinity of $k L=10.5$ in Figs. 2(c) and 2(d)], we obtain well pronounced asymmetry in transmission and unidirectional splitting in the inverse regime, when the defect layer is close to the exit interface. For instance, $T^{\leftarrow}=0.92, t_{0} \approx 0.17$ at $k L=9.54$ and $T^{\leftarrow}=0.88$, $t_{0} \approx 0.05$ at $k L=9.66$, both in Fig. 4(a), and $T^{\leftarrow}=0.8, t_{0}$ $=1.4 \times 10^{-2}$ at $k L=9.58$ in Fig. 4(b). Multiple Fabry-Perot type peaks of $T^{\leftarrow}$ with the main contribution of the \pm 1 -orders, which are observed in Fig. 4, indicate similarities of the resulting mechanism to that studied in Ref. 8.

In the range 4 , better suppression of zero order and splitting can be achieved for different locations of the defect layer. In the general case, splitting occurs for the both incidence directions, whereas $T^{\rightarrow} \neq T^{\leftarrow}\left(T^{\rightarrow}>0, T^{\leftarrow}>0\right)$, except for some peculiar values of $k L$. In Fig. 4 , it is seen that strong transmission (e.g., $T^{\leftarrow}>0$.9) can be obtained in the range 4 at the lower-side illumination, even if $t_{0} \approx 0$. This occurs, for example, at $k L=11.95$ in Fig. 4(b) and at $k L=11.2$ in Fig. 4(c). Two maxima of the nearly perfect

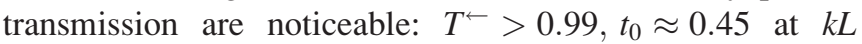
$=10.4$ in Fig. 4(a) and $T^{\leftarrow}>0.95, t_{0} \approx 0.48$ at $k L=10.37$ in Fig. 4(b). However, contribution of $t_{0}$ is not vanishing in these cases. Note that the number and location of the maxima of $t_{-1}^{\leftarrow}$ and $T^{\leftarrow}$ in the ranges 2 and 4 can also depend on location of the defect layer, see Fig. 4. The possible reason is that a thicker PhC segment (segment $C$ ) can create FabryPerot type maxima located denser than those created by a thinner one (segment $A$ ). In the other cases, transmission can be stronger at the upper-side illumination, e.g., $T \rightarrow=0.69$ at $k L=11.36$ in Fig. 2(a), $T^{\rightarrow}=0.82$ at $k L=11.45$ in Fig. 2(c) whereas $T^{\leftarrow} \approx 0$, and $T^{\rightarrow}=0.88$ at $k L=11.32$ in Fig. 2(d) whereas $T^{\leftarrow}=t_{0} \approx 0.03$.

To further demonstrate asymmetry in transmission, Fig. 5 presents the contrast $\sigma=20 \log _{10}\left(T^{\rightarrow} / T^{\leftarrow}\right)$ and $t_{-1}^{\rightarrow}$ together with $t_{-1}^{\leftarrow}$ for the range 4. In Fig. 5(a), it is seen that the contrast, a quantity connected with unidirectionality of splitting, is high in the ranges 1 and 3, also at a rather deep embedding of the defect layer. However, a suitable value of $\sigma$ can correspond to $\max \left(T^{\rightarrow}, T^{\leftarrow}\right)$ being not large enough. This situation is typical for the direct regime in the range 1. In the inverse regime in the range 2 , one can obtain $|\sigma|$ $>20 \mathrm{~dB}$, at least if the defect layer is not deeply embedded. A proper combination of transmission efficiency and contrast can be obtained in the range 3. However, its practical use for unidirectional splitting is again restricted to the cases when the embedding is not deep. The specifics and potential of the range 4 in splitting are determined by the fact that strong diffractions can appear at the both incidence directions. Figure 5(b) illustrates that the real bidirectional splitting, i.e., with $\eta=t_{-1}^{\rightarrow}=\overrightarrow{t_{+1}}=t_{-1}^{\leftarrow}=t_{+1}^{\rightarrow}(\eta=0.4$ at $k L=11.62)$ and unidirectional splitting in the inverse regime with $t_{-1}^{\leftarrow} \gg t_{-1}^{\rightarrow}$ (near $k L=11.95,|\sigma|=116$ ) can be obtained in one structure and, furthermore, at the close frequencies which correspond
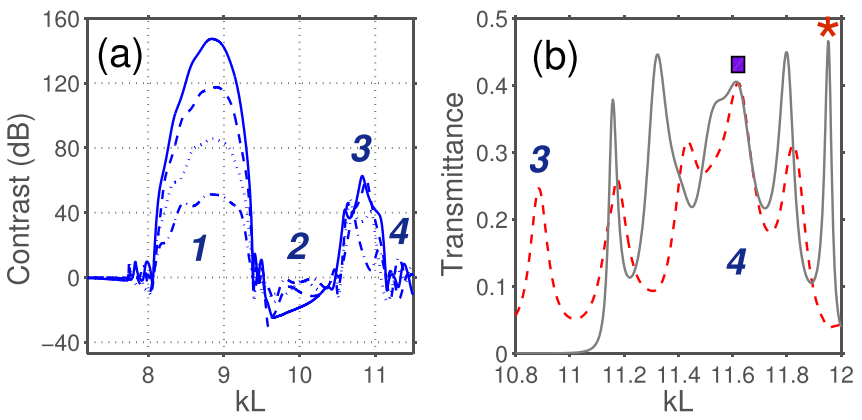

FIG. 5. (a) Contrast $\sigma=20 \log _{10}\left(T^{\rightarrow} / T^{\leftarrow}\right)$ for the structures in Figs. 2 and 4 when defect layer is second (solid line), third (dashed line), fourth (dotted line), and fifth (dash-dotted line) layer from the upper interface; (b) $\overrightarrow{t_{-1}}$ dashed red line and $t_{-1}^{\leftarrow}$ solid gray line, for the structures with defect layer being the third one from the upper interface, as in Figs. 2(b) and 4(b); rectangle and asterisk indicate the cases with $t_{-1}^{\rightarrow}=t_{-1}^{\leftarrow}$ and $t_{-1}^{\leftarrow} / t_{-1}^{\rightarrow} \approx 10$, respectively. 
to the same Floquet-Bloch mode. Note that bidirectional splitting with $0.3<\eta<0.4$ is also observed in Figs. 2(c), 2(d), 4(c), and 4(d), i.e., at different locations of the defect layer. The same remains true regarding the inverse regime of unidirectional splitting. However, the real bidirectionality case illustrated in Fig. 5(b) at $k L=11.62$ seems more useful for practical purposes. Since $t_{ \pm 1}=t_{ \pm 1}^{\leftarrow}$, a symmetric grating is mimicked and lack of symmetry is masked in this case.

\section{B. Varying structural parameters}

In line with the goals of this study, we demonstrate now generality of the effects observed in Figs. 2-5. Adjusting the material and geometrical parameters, one can obtain a nearly perfect unidirectional transmission, while zero order does not contribute to transmission. An example is presented in Fig. 6, where transmittance is shown at both lower-side and upper-side illumination for the structure which differs from that in Figs. 2 and 4 just in the rod material. The value of $\varepsilon=$ 11.4 is chosen here, which corresponds to the $\varepsilon$-range, to which $\mathrm{Si}, \mathrm{GaAs}$, and other widely used materials do belong. Comparing to the ranges 1 and 3 in Figs. 2 and 4, we obtain now higher efficiency, i.e., $T^{\rightarrow}=t_{-1}+t_{+1}>0.95$ at $k L=7$, whereas $T^{\leftarrow} \approx 0$ in a wide $k L$-range. Note that coupling to all of the propagating orders, i.e., $m=0, \pm 1$ is formally allowed at $k L>7.05$ by the EFCs (not shown). The peak of $T^{\rightarrow}=t_{0}+\overrightarrow{t_{-1}}+\overrightarrow{t_{+1}}>0.99$ at $k L=7.16$ [the highest peak in Fig. 6(a)] should also be noticed. However, it does not allow obtaining a nearly perfect splitting, since $t_{0} \approx 0.15$.

Next, we consider a structure with thinner rods that are made of material with $\varepsilon=9.61\left(\mathrm{Al}_{2} \mathrm{O}_{3}\right.$ below $\left.2 \mathrm{THz}\right)$, at upper-side illumination and the corresponding case of lowerside illumination, see Figs. 7 and 8, respectively. There are three typical $k L$-ranges denoted by 2,3 , and 4 , which are similar to the ranges denoted by the same numbers in Figs. 2-6. Indeed, zero order can be coupled to a Floquet-Bloch wave of the $\mathrm{PhC}$ in the ranges 2 and 4 , whereas only the \pm 1 orders can be coupled in the range 3 due to the defect layer with period $P=L=2 a$. Alongside the similarity of the ranges 3 and 4 to the corresponding ranges in Figs. 2-6, unusual behavior of transmission in the range 2 should be noticed. Therein, zero order can be well suppressed in
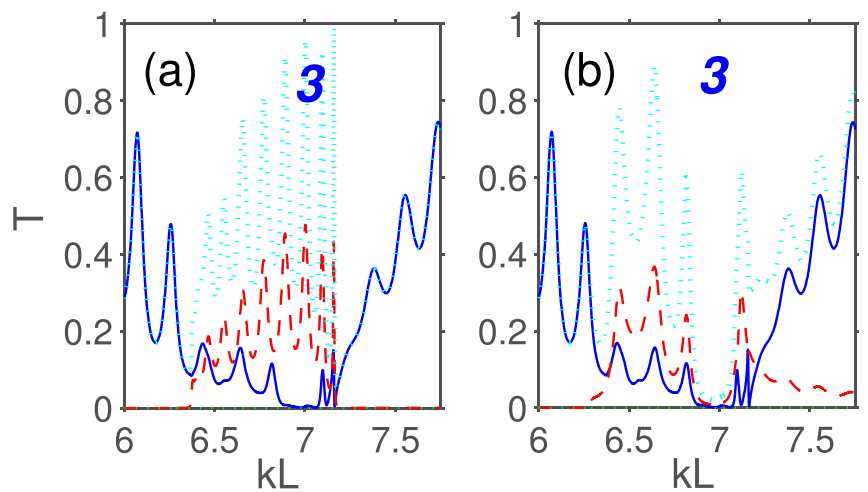

FIG. 6. Transmittance vs $k L$ for structure with defect layer being the second layer from the upper interface at (a) upper-side and (b) lower-side incidence; $d / a=0.4, \varepsilon=11.4$; solid blue line $t_{0}$, dashed red line (a) $t_{-1}=\overrightarrow{t_{+1}}$ and (b) $t_{-1}^{\leftarrow}=t_{+1}^{\leftarrow}$, dotted cyan line (a) $T^{\rightarrow}$ and (b) $T^{\leftarrow}$.
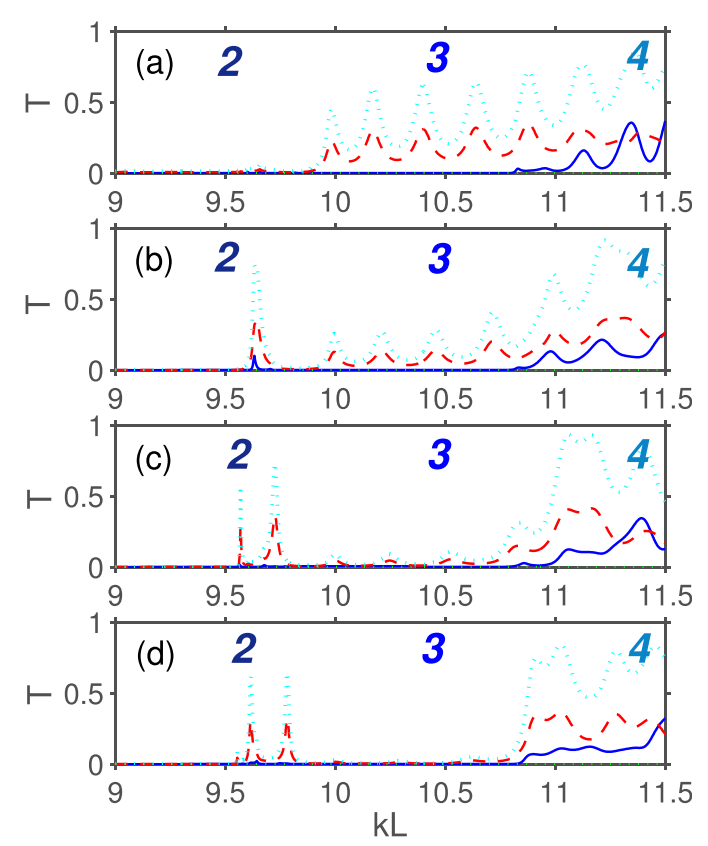

FIG. 7. Transmittance vs $k L$ for structure with defect layer being (a) second, (b) third, (c) fourth, and (d) fifth one from the upper (here incidence) interface; $d / a=0.3, \varepsilon=9.61$; solid blue line $t_{0}$, dashed red line $\overrightarrow{t_{-1}}$, and $\overrightarrow{t_{+1}}$, dotted cyan line $T^{\rightarrow}$.

transmission, in spite of being in-coupled. In this range, $T^{\rightarrow} \approx 0$ in Fig. 7(a), while $T^{\leftarrow}>0.5$ at the maxima due to the \pm 1 -orders in Fig. 8(a).

The effect of location of the defect layer strongly differs from one range to another. It is expected to depend on the properties of the corresponding Floquet-Bloch wave(s) and in-coupling conditions. For instance, if zero order may be in-coupled, location of the defect layer does not affect the principal possibility of splitting, except for the range 2 in
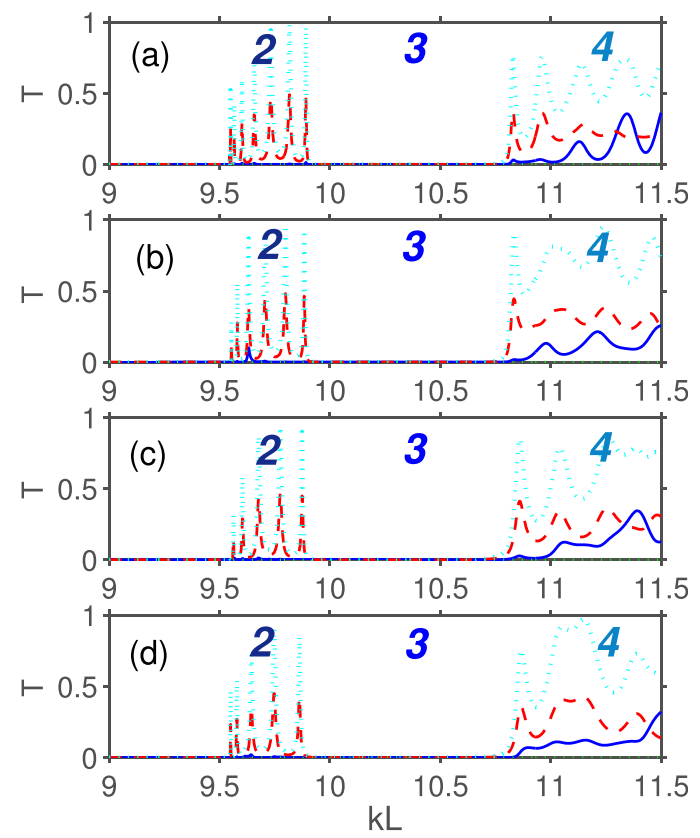

FIG. 8. Transmittance vs $k L$ for structure with defect layer being (a) second, (b) third, (c) fourth, and (d) fifth from the upper (here exit) interface; $d / a=0.3, \varepsilon=9.61$; solid blue line $t_{0}$, dashed red line $t_{-1}^{\leftarrow}$ and $t_{+1}^{\leftarrow}$, dotted cyan line $T^{\leftarrow}$. 
Fig. 7(a), where transmission is suppressed. On the contrary, if zero order may not be coupled, but the incidence interface cannot itself create the \pm 1 -orders, efficient tunneling through the segment $A$ would be necessary. In this case, the defect layer should transform evanescent waves into the higher-order waves propagating in the segment $C$. However, as seen in Fig. 7(d), four regular layers located between the incidence interface and the defect layer can be sufficient to suppress evanescent waves so that $T \rightarrow \approx 0$ in the range 3 . At lower-side illumination, transmittance in the ranges 2,3 , and 4 in Fig. 8 behaves similar to the ranges denoted with the same numbers in Fig. 4. The only significant difference is probably that $t_{0}$ is suppressed in the entire range 2 in Figs. 7(a) and 8(a) and, since $t_{0}^{\rightarrow}=t_{0}^{\leftarrow}$, splitting with $T^{\leftarrow}$ $=t_{-1}^{\leftarrow}+t_{+1}^{\leftarrow}$ can be obtained while $T^{\rightarrow} \approx 0$. At some maxima of $T^{\leftarrow}$, the defect layer works like a nearly perfect order convertor.

Figure 9 presents $t_{-1}^{\rightarrow}$ and $t_{-1}^{\leftarrow}$ at $9.4<k L<10$ for two structures from Figs. 7 and 8. In Fig. 9(a), there are three maxima of $t_{-1}^{\leftarrow}>0.45$, at which $\overrightarrow{t_{-1}}<0.01$ (denoted by asterisks). For one of them, $t_{-1}^{\leftarrow} \approx 0.49$ (near $k L=9.82$ ), that is closest to the ideal unidirectional splitting. Changing location of the defect layer, one can obtain different combinations of splitting regimes in one structure. For example, in Fig. 9(b) one can see that splitting with the features typical for asymmetric transmission in the inverse and the direct regime can appear at close frequencies, although transmission efficiency and contrast remain a subject of optimization. In particular, $t_{-1}^{\leftarrow}=0.45$ and $t_{-1}^{\hookrightarrow}=0.05$ near $k L=9.75, t_{-1}^{\rightarrow}$ $=0.32$ and $t_{-1}^{\leftarrow}=3.6 \times 10^{-2}$ near $k L=9.78$, and $t_{-1}^{\leftarrow}=0.43$ and $t_{-1}=7 \times 10^{-3}$ near $k L=9.86$ (denoted by asterisks). Such regimes can be important for obtaining of multifunctional operation that might include regimes with the waves guided by the defect layer, ${ }^{33}$ for which this layer should be located quite far from the incidence interface, like in Fig. 9(b). Note that a similar combination of unidirectional splitting regimes that would utilize one of the Floquet-Bloch modes as in the case considered has not yet been found in the nonsymmetric structures with one-side corrugations, e.g., see Ref. 8. It is noteworthy that combination of the direct and inverse regimes of unidirectional splitting can also be obtained in one structure in Figs. 7(b) and 8(b), and in Figs. 7(c) and 8(c). Besides, the real bidirectional splitting
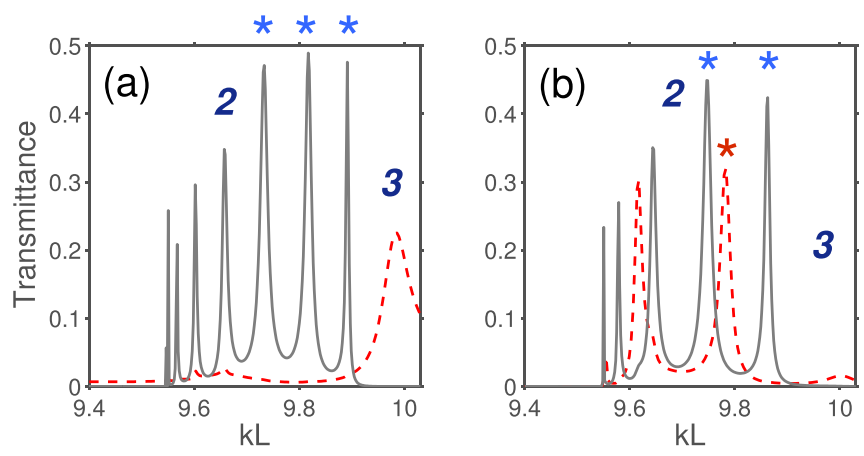

FIG. 9. $t_{-1}^{\rightarrow}$ (dashed red line) and $t_{-1}^{\leftarrow}$ (solid gray line) for (a) structure from Figs. 7(a) and 8(a) and (b) structure from Figs. 7(d) and 8(d); asterisks indicate selected cases with $\max \left(t_{-1}^{\leftarrow} / t^{\rightarrow}, t_{-1} / t_{-1}^{\leftarrow}\right)>8$. like that discussed in Fig. 5(b) can be obtained in the range 4 for the structures in Figs. 7(a)-7(d) and 8(a)-8(d). However, the case in Fig. 5(b) can be more appropriate because of higher efficiency and a peculiar combination of splitting regimes.

An example demonstrating that even a weak variation in $d / a$ may lead to strong differences in transmission is presented in Fig. 10, where a bit larger value of $d / a$ than in Figs. $7-9$ is used. The ranges are numbered in the same manner as in Figs. 2-8. In particular, one should notice a higher efficiency within the range 3 , where $T^{\rightarrow}=t_{-1}+t_{+1}$ and $T^{\leftarrow} \approx 0$. For instance, $T^{\rightarrow}=0.92$ at $k L=10.05$ and $T^{\rightarrow}=$ 0.89 at $k L=10.25$ in Fig. 10(a). Hence, performance of unidirectional direct-regime splitters can be enhanced by simple parameter adjustment. Moreover, the possibility of unidirectional inverse-regime splitting in the range 2 remains. For example, $T^{\leftarrow}=t_{-1}^{\leftarrow}+t_{+1}^{\leftarrow}=0.95$ and $T^{\rightarrow} \approx 1.1 \times 10^{-2}$ near $k L=9.35$ in Figs. 10(a) and 10(b). Comparing Fig. 10(a) with Fig. 10(b) and Fig. 10(c) with Fig. 10(d), one can see that the shift of the defect layer one period lower is still appropriate for the use of the range 3 for unidirectional splitting. On the contrary, the range 1 is not useful at all because of low efficiency, even if the defect layer is the second upper one, as in Fig. 10(a). The transmission features in the range 2 are rather similar to those in the same range in Figs. 2 and 4. It is worth noting that now the range 4 is less useful, since zero order contributes stronger than in the previous examples. The above discussed possibility of efficient suppression of zero order without utilizing the blocking effect connected with the specific EFC features and possibility of combining direct and inverse regimes in such a manner like in Fig. 9(b) enables more flexibility, on the one hand, but imposes restrictions on fabrication accuracy, on the other hand.

Finally, we demonstrate a way to wideband unidirectional splitting, at which transmission remains strong between the neighboring maxima. In fact, it exploits the merging of the maxima with the dominant contribution of higher orders. It can be achieved in the structures that are
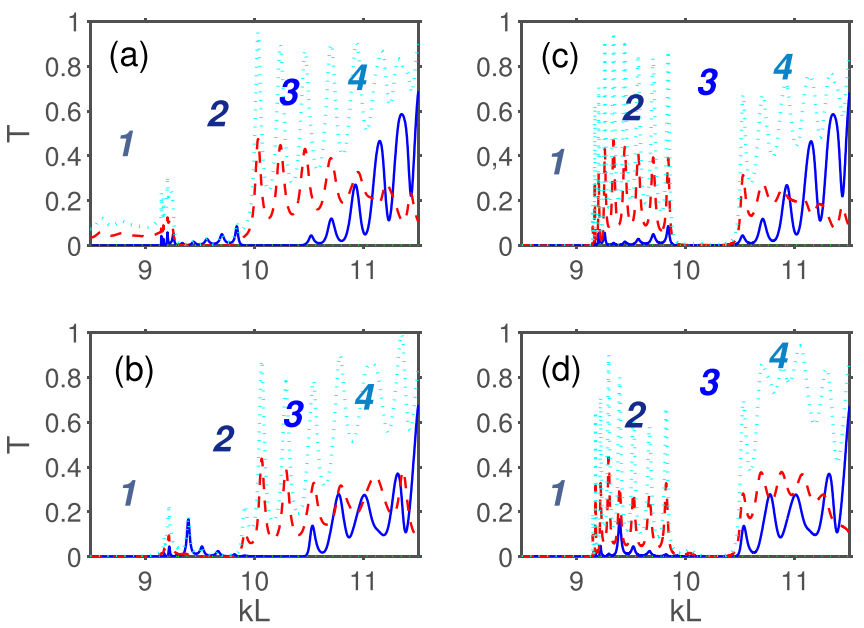

FIG. 10. Transmittance vs $k L$ for structure with defect layer being (a) second and (b) third layer from the upper incidence interface, and (c) second and (d) third layer from the upper exit interface; $d / a=0.32, \varepsilon=9.61$; solid blue line $t_{0}$; dashed red line (a) and (b) $t_{-1}^{\rightarrow}$ and $t_{+1}$, and (c) and (d) $t_{-1}^{\leftarrow}$ and $t_{+1}^{\leftarrow}$; dotted cyan line (a) and (b) $T^{\rightarrow}$ and (c) and (d) $T^{\leftarrow}$. 

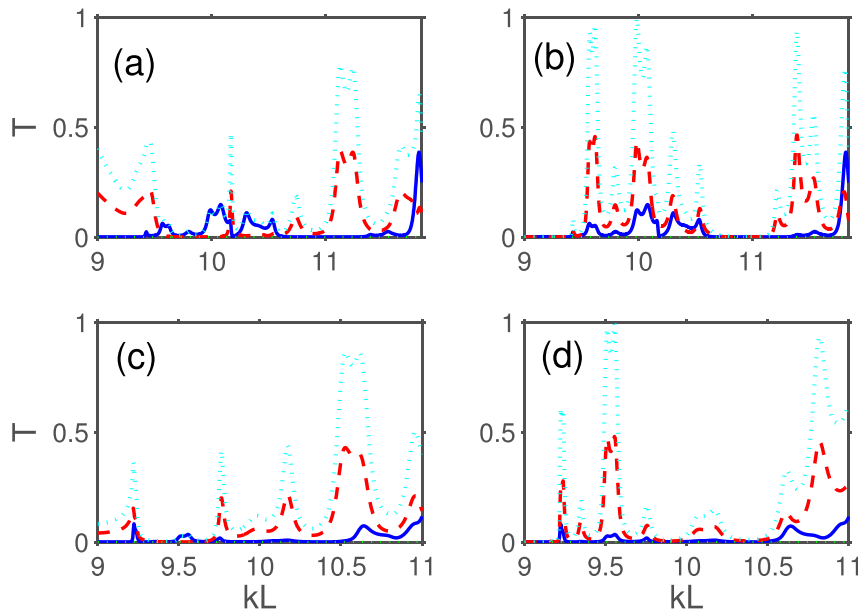

FIG. 11. Transmittance vs $k L$ for two structures with coupled-cavity type defect layer being (a) and (c) second layer from the upper incidence interface and (b) and (d) second layer from the upper exit interface, and additional line defect that is sixth layer from (a) and (c) the upper incidence interface and (b) and (d) the upper exit interface; (a) and (b) $d / a=0.4, \varepsilon=$ 5.8 and (c) and (d) $d / a=0.32, \varepsilon=9.61$; solid blue line $t_{0}$, dashed red line (a) and (c) $t_{-1}^{\rightarrow}=\underset{+1}{\overrightarrow{ }}$ and (b) and (d) $t_{-1}^{\leftarrow}=t_{+1}^{\leftarrow}$, dotted cyan line (a) and (c) $T^{\rightarrow}$ and (b) and (d) $T^{\leftarrow}$.

similar to those in Figs. 2, 4, 7, and 8 but modified by removing one layer of the rods from the segment $C$. Figure 11 presents transmittance in the case when the layer that is the sixth one from the upper interface is removed so that there are more six regular layers of the rods below the new line defect. In Fig. 11(a), a desired band with $T^{\rightarrow}=\overrightarrow{t_{-1}}+t_{+1}>$ 0.65 and $T^{\leftarrow} \approx 0$ is located around $k L=11.2$. Two wide bands with $t_{-1}^{\leftarrow}+t_{+1}^{\leftarrow}$ exceeding 0.78 and 0.62 are obtained in the inverse regime in Fig. 11(b) near $k L=9.6$ and $k L=10$, respectively. However, $\max t_{0}$ is equal to 0.06 and 0.15 for these bands, i.e., unidirectionality is imperfect. (Note that in Fig. 11(b) we obtain $T^{\leftarrow}>0.99$ at $k L=9.99$ ). Hence, one should find a compromise between the extent to which unidirectionality is pronounced and number of the bands suitable for wideband splitting. Wideband unidirectional splitting can be obtained in the direct regime also for the second performance, see Fig. 11(c). In this case, only the lowerfrequency part of the band located around $k L=10.6$ is unidirectional, i.e., $t_{0} \approx 0$ and $T^{\leftarrow} \approx 0$, although all of the orders $m=0$ and $m= \pm 1$ may formally contribute to $T^{\rightarrow}$. Wideband splitting observed in the inverse regime in Fig. $11(\mathrm{~d})$ at $k L=9.53$ seems to be a compromise between
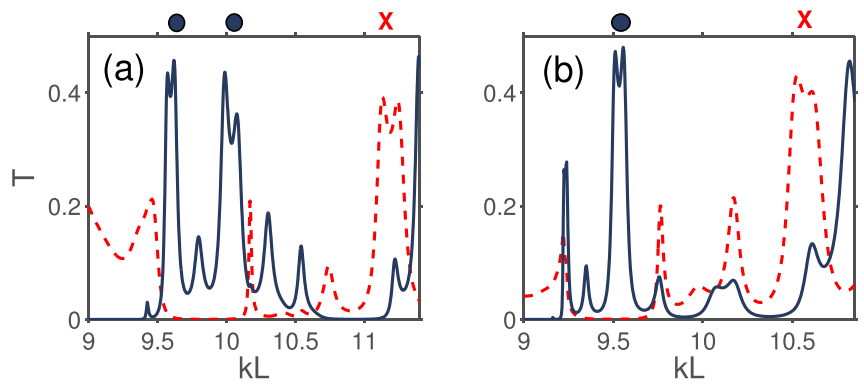

FIG. 12. $t_{-1}^{\rightarrow}$ (red dashed line) and $t_{-1}^{\leftarrow}$ (navy solid line) for the structures from (a) Figs. 11(a) and 11(b), and (b) Figs. 11(c) and 11(d); circles and crosses indicate the cases of wideband splitting that is connected with inverse and direct unidirectional transmission regimes, respectively. efficiency and strength of unidirectionality in terms of $\max \left(T^{\leftarrow} / t_{0}\right)$ and $\max \left(T^{\leftarrow /} / T^{\rightarrow}\right)$. To further characterize asymmetry in transmission at wideband splitting in Fig. 11, Fig. 12 presents $t_{-1}^{\rightarrow}$ and $t_{-1}^{\leftarrow}$ at upper-side and lower-side illumination in one plot, for each of the two performances from Fig. 11. Possibility of better suppression of zero order, which is allowed to be coupled, and main features of the band widening mechanism that is based on twinning and merging of the transmission maxima invoke a more detailed study.

\section{CONCLUSION}

To summarize, we studied the potential of photonic crystal (PhC) based structures, whose spatial inversion symmetry is broken by using a periodic defect layer, in diffraction inspired dual-beam splitting. We demonstrated what and how can be achieved and what might be difficult to achieve for this class of the structures. The basic splitting mechanisms that have earlier been studied in the nonsymmetric $\mathrm{PhC}$ based structures with interface corrugations are obtained in this paper for similar photonic structures that do not have external corrugations. Various regimes of unidirectional and bidirectional splitting have been discussed. It is shown that zero order can be well suppressed even if its coupling to a Floquet-Bloch mode of the $\mathrm{PhC}$ is allowed by the dispersion. Moreover, it can be the only order that contributes to incoupling, whereas higher orders required for a propagatingwave regime are created by the energy redistribution at the embedded defect layer. Thus, unidirectional splitting, whose existence has often been associated with blocking of zeroorder transmission owing to the specific dispersion properties, is a general regime obtainable at different combinations of dispersion, material, and geometrical characteristics. Furthermore, strong higher-order transmission and related splitting can appear for two opposite incidence directions in the neighboring frequency ranges, depending on dispersion and coupling conditions. As follows from the obtained results, the defect layer should be located not far from one of the interfaces in order to obtain unidirectional splitting in the direct regime. In the inverse regime, it can be achieved also for a deeply embedded defect layer. This feature and high capability of integration with other devices on the PhC platform are very important for future applications of the structures with the embedded defects in multifunctional and reconfigurable devices, in which the same defect layer may work, for instance, like a waveguide in another frequency range in the same structure.

Compared to the earlier studies of the nonsymmetric structures with one-side external corrugations, some peculiar regimes may show even better characteristics. Clearly, this does not mean that the structures with external corrugations may not show better characteristics in principle but rather indicates that not all of them have already been found. In particular, this is related to the inverse-regime dual-beam unidirectional splitting and reversing direction of highefficiency transmission at unidirectional splitting for weak frequency variations. It is noteworthy that bidirectional splitting can be achieved at the deep embedding of the defect layer. In particular, the real bidirectional splitting should be 
mentioned, at which transmission and diffraction at some frequencies are exactly the same for two opposite incidence directions, in spite of that the structure is nonsymmetric with respect to the midplane $(y=D / 2, D$ is the thickness of the entire slab). In the other words, the lack of symmetry is not recognizable in the transmittance data. The obtained results indicate a route to future multifunctional devices and related directions of perspective research. Since the dielectric materials, which are suggested for the use, are low-loss and weakly dispersive in a wide frequency range including microwave and optical frequencies, the studied structures should be rescalable within this range. Possible realization of the suggested approach with the aid of metamaterials will be a subject of one of the next studies.

\section{ACKNOWLEDGMENTS}

This work is supported by the Projects DPT-HAMIT, ESF-EPIGRAT, and NATO-SET-181, and by TUBITAK under the Project Nos. 107A004, 109A015, and 109E301. A.E.S. thanks TUBITAK for partial support in the framework of the Visiting Researcher Program and National Science Centre, Poland for financial assistance under the Projects MagnoWa DEC-2-12/07/E/ST3/00538, and MetaSel DEC-2015/17/B/ST3/00118. E.O. acknowledges partial support from the Turkish Academy of Sciences.

${ }^{1}$ A. Figotin and I. Vitebskiy, Phys. Rev. B 67, 165210 (2003).

${ }^{2}$ F. D. M. Haldane and S. Raghu, Phys. Rev. Lett. 100, 013904 (2008).

${ }^{3}$ M. Schmitz, R. Bräuer, and O. Bryngdahl, Opt. Lett. 20, 1830 (1995).

${ }^{4}$ Ph. Lalanne, J. Hazart, P. Chavel, E. Cambril, and H. Launois, J. Opt. A: Pure Appl. Opt. 1, 215 (1999).

${ }^{5}$ J. Feng and Z. Zhou, Opt. Lett. 32, 1662 (2007).

${ }^{6}$ E. Colak, A. E. Serebryannikov, A. O. Cakmak, and E. Ozbay, Appl. Phys. Lett. 102, 151105 (2013).

${ }^{7}$ A. E. Serebryannikov, Phys. Rev. B 80, 155117 (2009).

${ }^{8}$ A. E. Serebryannikov, K. B. Alici, T. Magath, E. Colak, and E. Ozbay, Phys. Rev. A 86, 053835 (2012).

${ }^{9}$ S. Xu, C. Qiu, and Z. Liu, J. Appl. Phys. 111, 094505 (2012).

${ }^{10}$ A. Mandatori, M. Bertolotti, and C. Sibilia, J. Opt. Soc. Am. B 24, 685 (2007).
${ }^{11}$ X.-F. Li, X. Ni, L. Feng, M.-H. Lu, C. He, and Y.-F. Chen, Phys. Rev. Lett. 106, 084301 (2011).

${ }^{12}$ R. Singh, E. Plum, C. Menzel, C. Rockstuhl, A. K. Azad, R. A. Cheville, F. Lederer, W. Zhang, and N. I. Zheludev, Phys. Rev. B 80, 153104 (2009).

${ }^{13}$ J. H. Shi, H. F. Ma, C. Y. Guan, Z. P. Wang, and T. J. Cui, Phys. Rev. B 89, 165128 (2014).

${ }^{14}$ Y. Xu, Q. Shi, Z. Zhu, and J. Shi, Opt. Express 22, 25679 (2014).

${ }^{15}$ M. Mutlu, S. Cakmakyapan, A. E. Serebryannikov, and E. Ozbay, Phys. Rev. B 87, 205123 (2013).

${ }^{16}$ C. Wang, X.-L. Zhong, and Z.-Y. Li, Sci. Rep. 2, 674 (2012).

${ }^{17}$ A. Cicek, O. A. Kaya, and B. Ulug, Appl. Phys. Lett. 100, 111905 (2012).

${ }^{18}$ F. T. Gundogdu, A. E. Serebryannikov, A. O. Cakmak, and E. Ozbay, Opt. Express 23, 24120 (2015).

${ }^{19}$ J. H. Oh, H. W. Kim, P. S. Ma, H. M. Seung, and Y. Y. Kim, Appl. Phys. Lett. 100, 213503 (2012).

${ }^{20}$ M. Beruete, A. E. Serebryannikov, V. Torres, M. Navarro-Cia, and M. Sorolla, Appl. Phys. Lett. 99, 154101 (2011).

${ }^{21}$ T. Xu and H. J. Lezec, Nat. Commun. 5, 4141 (2014).

${ }^{22}$ P. Rodriguez-Ulibarri, V. Pacheco-Pena, M. Navarro-Cia, A. E. Serebryannikov, and M. Beruete, Appl. Phys. Lett. 106, 061109 (2015).

${ }^{23}$ M. Stolarek, M. Yavorskiy, R. Kotynski, C. J. Zapata Rodriguez, J. Lusakowski, and T. Szoplik, Opt. Lett. 38, 839 (2013).

${ }^{24}$ S. Cakmakyapan, A. E. Serebryannikov, H. Caglayan, and E. Ozbay, Opt. Express 20, 26636 (2012).

${ }^{25}$ V. Kuzmiak and A. A. Maradudin, Phys. Rev. A 86, 043805 (2012).

${ }^{26}$ F. V. Liu, D. A. B. Miller, and S. Fan, Opt. Express 20, 28388 (2012).

${ }^{27}$ L. Feng, M. Ayache, J. Huang, Y.-L. Xu, M.-H. Lu, Y. F. Chen, Y. Fainman, and A. Scherer, Science 333, 729 (2011).

${ }^{28}$ N. Yu, P. Genevet, M. Kats, F. Aieta, J.-P. Tetienne, F. Capasso, and Z. Gaburro, Science 334(6054), 333 (2011).

${ }^{29}$ F. Aieta, P. Genevet, N. Yu, M. Kats, Z. Gaburro, and F. Capasso, Nano Lett. 12, 1702 (2012).

${ }^{30}$ C. Pfeiffer and A. Grbic, Phys. Rev. Lett. 110, 197401 (2013).

${ }^{31}$ A. Mehdipour, J. W. Wong, and G. V. Eleftheriades, IEEE Trans. Antennas. Propag. 63, 978 (2015).

${ }^{32}$ K. Sakoda, Optical Properties of Photonic Crystals (Springer, Berlin, 2001).

${ }^{33}$ Photonic Crystals. Physics, Fabrication and Applications, edited by K. Inoue and K. Othaka (Springer, Berlin, 2004).

${ }^{34}$ T. Magath and A. E. Serebryannikov, J. Opt. Soc. Am. A 22, 2405 (2005).

${ }^{35}$ See www.cst.com for software details.

${ }^{36}$ R. Petit, Electromagnetic Theory of Gratings (Springer, Berlin, 1980).

${ }^{37}$ S. Foteinopoulou and C. M. Soukoulis, Phys. Rev. B 72, 165112 (2005). 